\title{
Create the Individualized Digital Twin for Noninvasive Precise Pulmonary Healthcare
}

\author{
Yu Feng ${ }^{1 *}$, Xiaole Chen ${ }^{2}$ and Jianan Zhao ${ }^{1}$ \\ ${ }^{1}$ School of Chemical Engineering, Oklahoma State University, USA \\ ${ }^{2}$ School of Energy and Environment, Southeast University, China \\ *Corresponding author: Yu Feng, School of Chemical Engineering, Oklahoma State University, Stillwater, OK, USA \\ Submission: December 16, 2017; Published: January 19, 2018
}

Abstract

Nowadays, "personalized medicine" is starting to replace the current "one size fits all" approach.It is crucial to understand the aerosol drug dynamics better and develop a revolutionary patient-specific pulmonary disease treatment platform and to improve therapeutic outcomes. In this paper, the discussion paves the way to achieve personalized pulmonary healthcare by introducing the digital twin and lung aerosol dynamics modeling platform as a noninvasive tool for individualized treatment planning. Challenges and breakthroughs are also discussed as the future research directions.

\section{Personalized Treatment Plan: Precision, Effectiveness, and Challenges}

(a) Conventional Drug Delivery Method

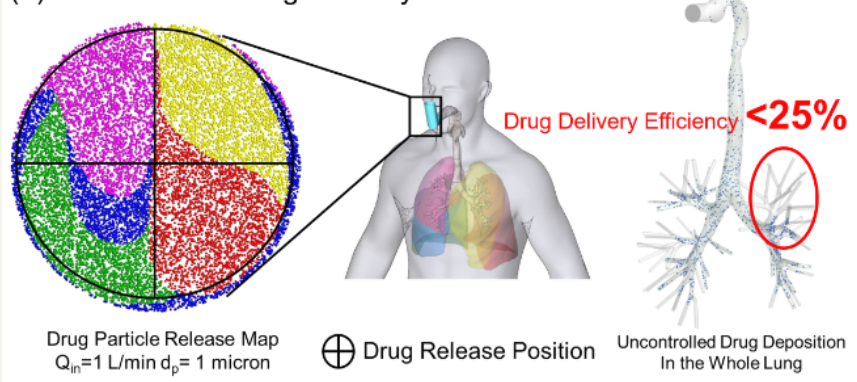

(b) "Controlled Air-Drug Stream" Targeting Method

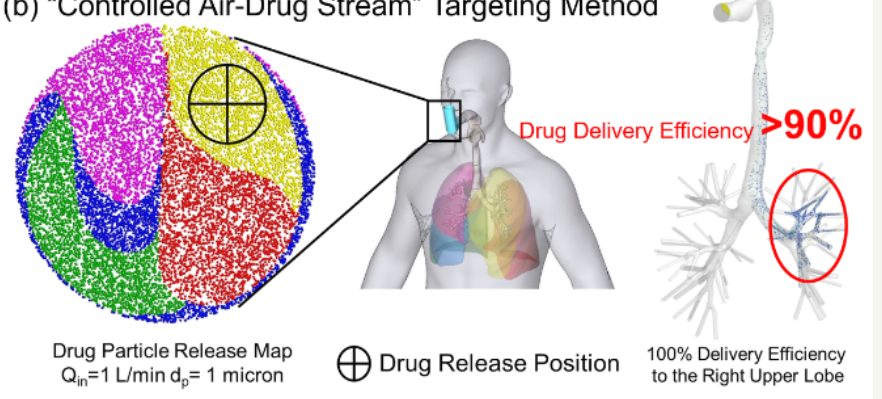

Figure 1: Targeting the right upper lobe using the "Controlled Air-Drug Stream Method in the Individualized Digital Twin Prototype: (a) Conventional Drug Inhalation Therapy $(<25 \%)$ and (b) the “Controlled Air-Drug Stream" Delivery Method ( $>90 \%)$.

Lung cancer, a highly fatal disease, killed 1.6 million of people in 2012 and accounted for billions in health care costs worldwide. Given the fact that the existing lung cancer treatments have major portions of the aggressive medicine deposit on healthy tissue, there is an urgent need to develop a revolutionary patient-specific pulmonary drug delivery method to effectively target localized lung tumors and thereby protect patients from undesired side effects, reduce the healthcare cost, as well as enhance the therapeutic outcomes. Enlightened by the idea of personalized health care and based on the trends that personalized medicine is starting to replace the current "one size fits all" approach to medical treatment, the goal is to develop an effective personalized pulmonary healthcare planning tool to deliver the right dose of the right drug at the right time and location to the specified patient with a generalized pulmonary drug delivery modality to treat lung cancer. An example of personalized pulmonary healthcare planning is the targeted pulmonary drug delivery methodology [1,2]. Indeed, conventional pulmonary drug delivery devices still have poor efficiencies $(<25 \%)$ for delivering drugs to the lung tumor sites. Major portions of the aggressive medicine deposit on healthy tissue (Figure 1a). In contrast, by designing a patient-specific targeted drug delivery method (Figure 1b), clinical doctors will be able to increase the local deposition efficiency of drugs to $90 \%$. However, traditional in vitro and in vivo studies are limited and not sufficient for the personalized treatment plan development purpose. Specifically, due to the invasive nature and imaging limitations, animal studies and clinical tests are lack of operational flexibilities and will not be able to provide insightful high-resolution patient-specific data. Therefore, alternative methods should be developed to conquer these bottlenecks. Models based on the computational fluid-particle 
dynamics (CFPD) method play a critical role in exploring alternate study designs and provide high-resolution data in the noninvasive, cost-effective, and time-saving manner. The in silico methodologies can fill the knowledge gap due to the deficiency of traditional in vitro and in vivo methods, as well as make breakthroughs to pave the way to establish a reliable and efficient numerical investigation framework for pulmonary healthcare on a patient-specific level. Foreseeable challenges include [3]: (1) Reconstruction of a whole- lung configuration to enable to simulation of inspiration-expiration full breathing cycle on particle transport and deposition; (2) Inter-subject variability studies for a more statistically robust numerical analysis, i.e., CFPD simulation with "error bars"; and (3) Establishment of a multiscale model to bring the simulation from lung deposition to health endpoints, i.e., translocations in the whole body.

\section{Computational Fluid Particle Dynamics Models for Lung Aerosol Dynamics}

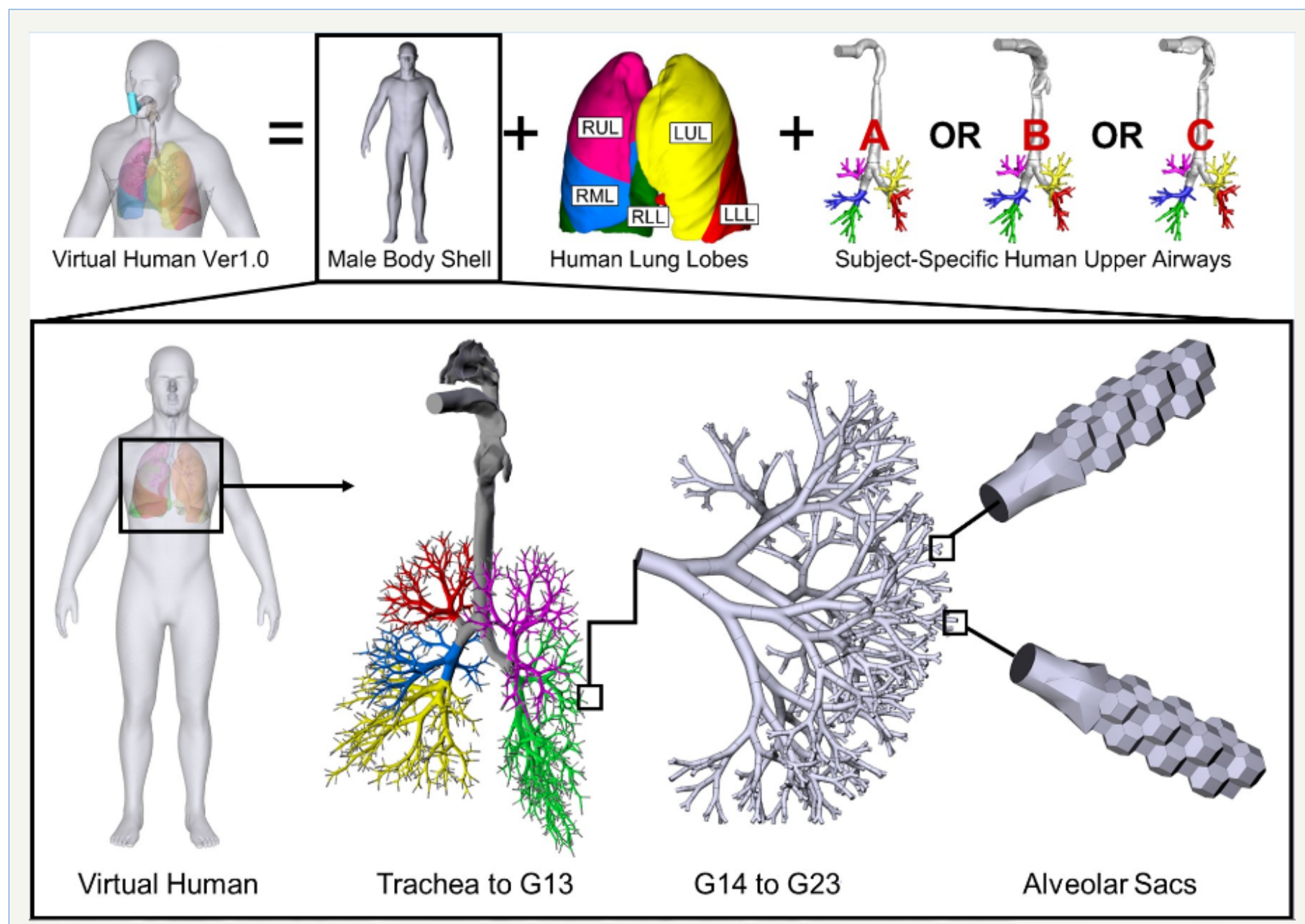

Figure 2: The CBBL virtual human system V1.0 with a representative human respiratory system for Computational FluidParticle Dynamics (CFPD) simulations covering the entire conducting and respiratory zones.

Focusing on advancing the frontier of computational lung aerosol dynamics, it is crucial to build the individualized digital twin and associated numerical investigation framework based on conservation laws, Computational Fluid-Particle Dynamics (CFPD) models can provide comprehensive analysis of inhaled drug particulate matter dynamicswith insightful answers to the key question for personalized treatment planning: "What type of drug particle deposits where at what surface concentrations in the representative whole-lung configuration under full inspiratoryexpiratory breathing cycles?" This enables safe and effective therapeutics to advance more efficiently through the different stages of clinical trials. "Specifically, a couple of breakthroughs have been made using the Euler-Euler, Euler-Lagrange, as well as the new Dense Discrete Phase Model (DDPM) with Discrete Element Method (DEM), on the virtual human system development with a whole-lung model (Figure 2), the inter-subject variability studies with the virtual population group (Figure 3), and the multiscale CFPD-PBPK/TK model development (Figure 4), by integrating the most advanced CFPD platforms and the most efficient speed-up on local workstations [4-7]. 

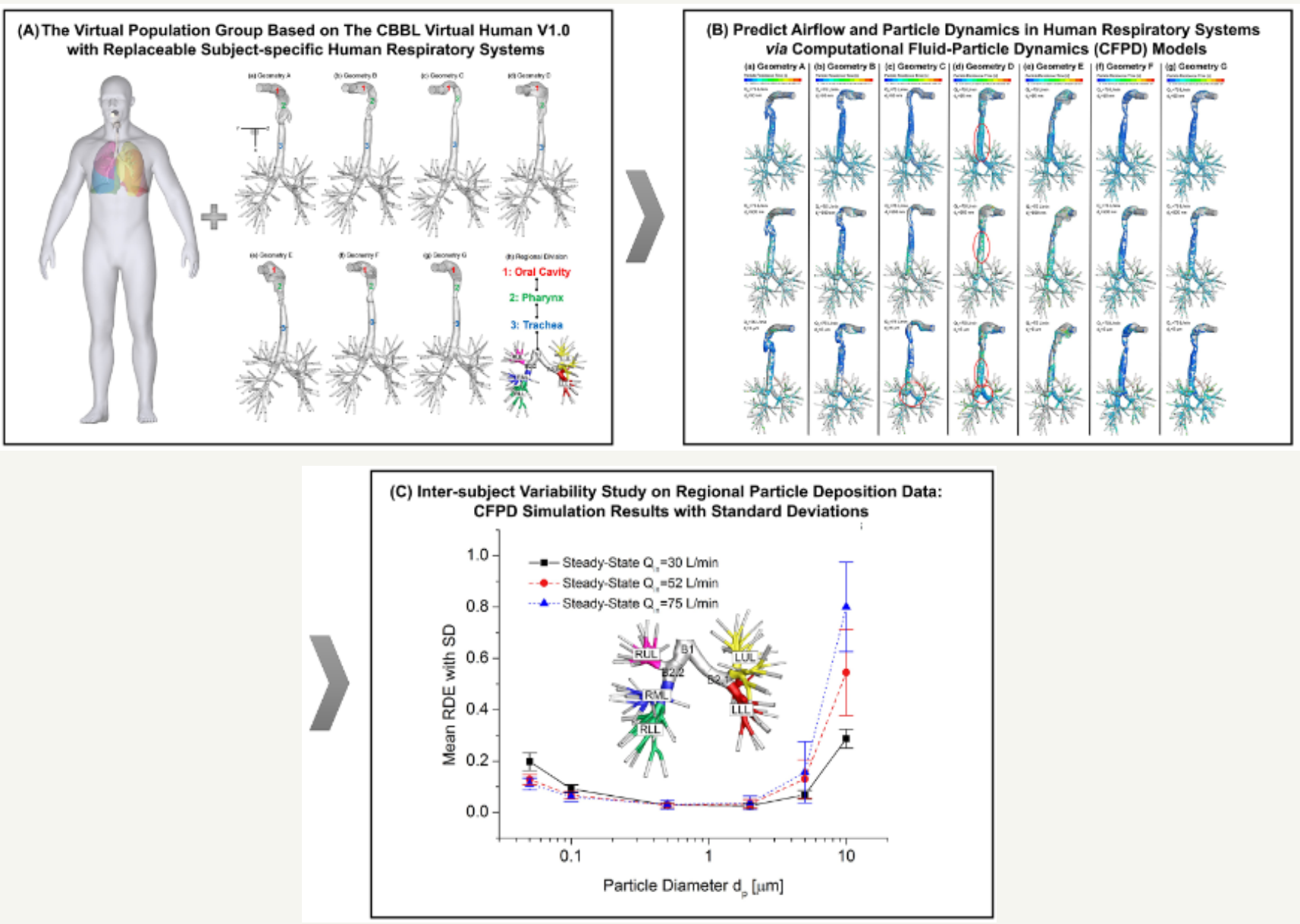

Figure 3: The CBBL Virtual Population Group V1.0 and the In-Silico Intersubject Variability Investigation Framework.

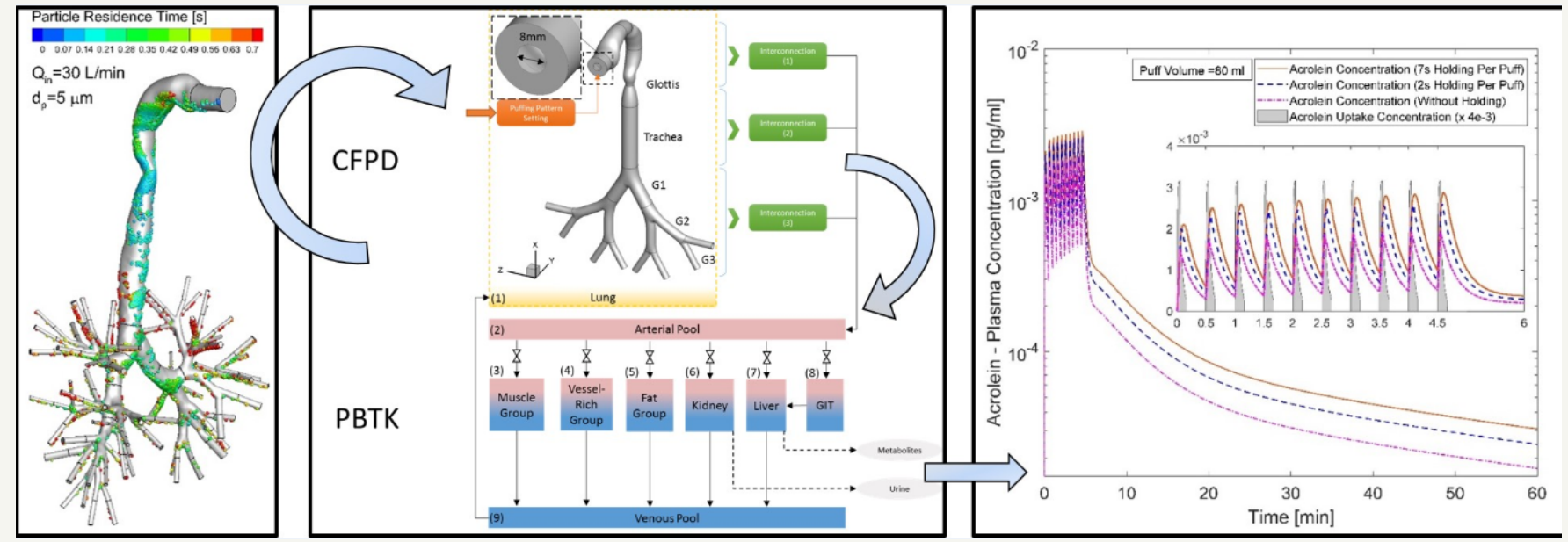

Figure 4: The Multiscale Computational Fluid-Particle Dynamics (CFPD) plus Physiological Based Toxicokinetic (PBTK) modeling framework.

The Virtual Human System V1.0: The Prototype of the Individualized Digital Twin

A new virtual human system, i.e., a standing 47 years old digital male with the high-resolution human respiratory system covering the entire conducting and respiratory zones, lung lobes, and body shell has been developed (Figure 2). Serving as the prototype of the "individualized digital twin," the virtual human V1.0 is CFPD ready, and the subject-specific body shell also enables the subject-specific health risk assessment for in-silico occupational exposure studies, including simulations of real-time ventilation, skin absorption, and lung deposition. The lung airways and body shells are replaceable, 
which will lead to the ultimate goal, i.e., the individualized digital twin, either standing or seating, male or female, adults or kids, with or without lung diseases. The in silico era for pulmonary health is around the corner.

\section{The Virtual Population Group V1.0: CFPD Simulation} Results with "Error Bars"

Numerical results [6] indicate that inter-subject variability effect on drug particle transport and deposition is significant Therefore, it is inevitable to improve the statistical robustness of the in silico study processfor lung aerosol dynamics. The Virtual Population Group (VPG) are a set of detailed high-resolution anatomical models created from CT/MRI data of human subjects (Figure 3). The development of such a VPG opens up for the first time the possibility to comprehensively analyze variations in the general population or for specific subpopulation groups, resulting in the enhancement of the statistical robustness of numerical studies. However, such analyses consider individual anatomical differences, and are thus computationally expensive. Therefore, precomputed lung aerosol dynamics libraries should be built, to simplify the personalized pulmonary drug delivery planning (Figure 5).

\section{The Multiscale CFPD-PBPK/TK Modeling Framework: To the Health Endpoints}

Lung deposition is not the health endpoints. Toxicologist, pharmacist, and clinical doctors are more interested in the afterdeposition dynamics, i.e., the time course of therapeutic or toxic species in plasma and different organs through the whole human body. One of the efforts is the development of a experimentally validated multiscale numerical model, i.e., Computational Fluid-
Particle Dynamics (CFPD) model combined with a Physiologically Based Toxicokinetic (PBTK) model [7] to predict the systemic translocation of nicotine and acrolein in the human body after the deposition in the respiratory system (Figure 4 for the modeling framework). These frame work enables simulations of extremely complex lung aerosol dynamics phenomena and whole-body translocation mechanisms at detailed levels never undertaken before. The framework can be easily modified to fit in other pulmonary research areas, such as drug delivery and occupational exposure risk assessment.

\section{The Future: Personalized Pulmonary Healthcare} Planner in an APP

Compared to conventional methods, in silico pulmonary healthcare planning platform is promising in a more accurate, noninvasive, and fast manner. In the near future, the workflow (Figure 5) of personalized lung disease treatment is approachable even in a single APP developed using software such as ANSYS ACT in Python which will conquer complex simulation needs. Clinical doctors without substantial background in lung aerosol dynamics can use the APP to design the treatment plan without any difficulties. Obtaining a few morphological parameters based on the patient-specific CT/MRI data of the human respiratory system configurations as well as the coordinates of the lesions, the personalized pulmonary healthcare planner will provide an integrated solution to target localized lung sites based on the pre-computed database connected with a reliable machinelearning model. It is fast, noninvasive, reliable, easy-to-use, and the best feature is that the planning is patient-specific with your individualized digital twin.

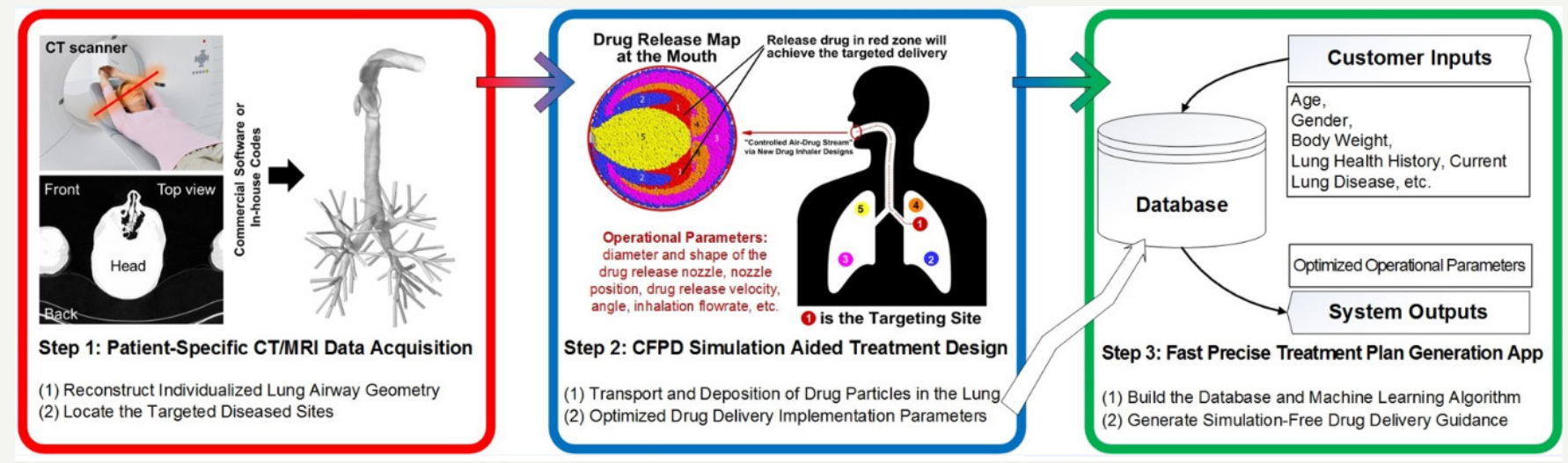

Figure 5: In silico clinical trial schematic and personalized targeted drug delivery planning platform.

\section{Acknowledgement}

The use of ANSYS software (Canonsburg, PA) as part of the ANSYS-OSU academic partnership agreement is gratefully acknowledged. Some of the computing for this project was performed at the OSU High Performance Computing Center at Oklahoma State University (Dr. Dana Brunson, Director and Dr. Evan Linde, Research Cyberintrastructure Analyst).

\section{References}

1. Feng Y (2017) A New Patient-Specific Pulmonary Drug Targeted Delivery Method to Treat Lung Cancer using E-Cigarette Technology. AIChE 2017 Annual Meeting, Minneapolis, MN, USA

2. Feng Y, Chen X, Xu Z, Haghnegahdar A (2017) Intersubject Variability in Pulmonary Drug Delivery Efficiency to Target Lung Tumors at Different Lobes: An In-Silico Study. BMES 2017 Annual Meeting, Phoenix, AZ, USA.

3. Feng Y, Xu Z, Haghnegahdar A (2016) Computational Fluid-Particle Dynamics Modeling for Unconventional Inhaled Aerosols in Human 
Respiratory Systems, Aerosols - Science and Case Studies. Volkov Konstantin (Ed.), InTech, DOI: 10.5772/65361.

4. Feng Y, Kleinstreuer C (2013) Analysis of non-spherical particle transport in complex internal shear flows. Physics of Fluids 25: 091904.

5. Feng Y, Kleinstreuer C (2014) Micron-particle transport, interactions and deposition in triple lung-airway bifurcations using a novel modeling approach. Journal of Aerosol Science 71: 1-15.
6. Feng Y, Kleinstreuer C, Wang J, Wu DH, Lin J (2018) An In-Silico Intersubject Variability Study of the Extrathoracic Morphology Effect on the Transport and Deposition of Inhaled Particles in the Tracheobronchial Tree. Journal of Aerosol Science.

7. Feng Y, Kleinstreuer C, Castro N, Rostamic A (2017) Computational Analysis of the Deposition and Translocation of Inhaled Nicotine and Acrolein in Human Body with E-cigarette Puffing Topographies. Aerosol Science and Technology 96(2016): 96-123. 